\title{
On coupled systems of Hammerstein integral equations
}

\author{
Robert de Sousa ${ }^{1 *}$ and Feliz Minhós ${ }^{2}$
}

\author{
"Correspondence: \\ robert.sousa@docente.unicv.edu.cv \\ 1 Faculdade de Ciências e \\ Tecnologia, Núcleo de Matemática \\ e Aplicações (NUMAT), Universidade \\ de Cabo Verde, Praia, Cabo Verde \\ Full list of author information is \\ available at the end of the article
}

\begin{abstract}
In this paper we deal with generalized coupled systems of integral equations of Hammerstein type with nonlinearities depending on several derivatives of both variables and we underline that both equations and both variables can have a different regularity. This detail is very important as it allows for the application to, for example, boundary value problems with coupled systems composed of differential equations of different orders and distinct boundary conditions. This issue will open a new field of applications to phenomena modeled by coupled systems requiring different types of regularity for the unknown functions.

The arguments follow Guo-Krasnosel'skii compression-expansion theory on cones and the kernel functions are nonnegative and verifying adequate sign and growth assumptions.

The dependence of the derivatives is overcome by the construction of suitable cones taking into account certain conditions of sublinearity/superlinearity at the origin and at $+\infty$.
\end{abstract}

MSC: 45G15; 34B15; 34B18; 47H30; 34B27

Keywords: Coupled systems; Hammerstein integral equations; $L^{\infty}$-Carathéodory and kernels functions; Guo-Krasnosel'skiĭ theorem in cone; Arzelà-Ascoli theorem

\section{Introduction}

In this paper we deal with generalized coupled systems of integral equations of Hammerstein type,

$$
\left\{\begin{array}{l}
u_{1}(t)=\int_{0}^{1} k_{1}(t, s) g_{1}(s) f_{1}\left(s, u_{1}(s), \ldots, u_{1}^{\left(m_{1}\right)}(s), u_{2}(s), \ldots, u_{2}^{\left(n_{1}\right)}(s)\right) d s \\
u_{2}(t)=\int_{0}^{1} k_{2}(t, s) g_{2}(s) f_{2}\left(s, u_{1}(s), \ldots, u_{1}^{\left(m_{2}\right)}(s), u_{2}(s), \ldots, u_{2}^{\left(n_{2}\right)}(s)\right) d s
\end{array}\right.
$$

where $k_{\iota}:[0,1]^{2} \rightarrow \mathbb{R}, \iota=1,2$, are the kernel functions such that $k_{\iota} \in W^{r_{\iota}, 1}\left([0,1]^{2}\right), r_{\iota}=$ $\max \left\{m_{\iota}, n_{\iota}\right\}$, with $m_{\iota}, n_{\iota} \geq 0$ positive integers, $g_{\iota} \in L^{1}([0,1])$ with $g_{\iota}(t) \geq 0$ for a.e. $t \in[0,1]$, and $f_{l}:[0,1] \times \mathbb{R}^{m_{l}+n_{l}+2} \rightarrow[0, \infty)$ are $L^{\infty}$-Carathéodory functions.

The theory of integral equations has been and continues to be a field of many research and applications. These equations are especially relevant in physics and are often used to reformulate or rewrite mathematical problems.

(c) The Author(s) 2019. This article is distributed under the terms of the Creative Commons Attribution 4.0 International License (http://creativecommons.org/licenses/by/4.0/), which permits unrestricted use, distribution, and reproduction in any medium, provided you give appropriate credit to the original author(s) and the source, provide a link to the Creative Commons license, and indicate if changes were made. 
Hammerstein integral equations are special subclasses of nonlinear integral equations of Fredholm-type

$$
u(x)=g(x)+\int_{a}^{b} k(x, y, u(x), u(y)) f(x, u(y)) d y, x \in[a, b]
$$

and their study was initiated by Hammerstein (see [4, 17]).

Hammerstein family of integral equations appears in some mathematical models, such as electrostatic drift waves and low-frequency electromagnetic perturbation (see [14]) and signal theory (see [19]). Other applications and very successful results can be found on [2, 10, 21] and the references therein.

The existence, uniqueness, multiplicity, positivity and location of solutions are the most studied and predominant elements as regards Hammerstein integral equations. Citing just a few examples in the literature, we mention [24], where the authors use fixed point index theory to establish their main result, based on a priori estimates achieved by nonnegative matrices; in [11], Coclite studies the existence of a positive measurable solution of the Hammerstein equation of the first kind with a singular nonlinear term at the origin; in [8], the authors contribute, by monotone iterative methods, combined with the classical fixed point index, proving two results concerning non-decreasing and non-increasing operators in a shell, in the presence of an upper or a lower solution; in [9], Cardinali et al., examine multivalued Hammerstein integral equations defined in a separable reflexive Banach space, obtaining existence results for convex and nonconvex problems; in [13], the researchers study solutions of the nonlinear Hammerstein integral equation with changingsign kernels by using a variational principle of Ricceri and critical points theory techniques (they combine the effects of a sublinear and superlinear nonlinear terms to establish new existence and multiplicity results); in [26], the authors study the existence and the uniqueness of iterative positive solutions for a class of nonlinear singular integral equations in which the nonlinear terms may be singular in both time and space variables. By using the fixed point theorem of mixed monotone operators in cones, they establish the conditions for the existence and uniqueness of positive solutions to the problem.

In addition, several discretization and numerical methods were also considered on integral equations (see, for instance, [1, 3, 5-7, 22, 25]).

More specifically, about Hammerstein-type coupled systems of integral equations, we refer [12], where, by a special cone and using fixed point index theory, Cui and Sun, investigate the existence of positive solutions of singular superlinear coupled integral boundary value problems for differential systems

$$
\left\{\begin{array}{l}
-x^{\prime \prime}(t)=f_{1}(t, x(t), y(t)) \\
-y^{\prime \prime}(t)=f_{2}(t, x(t), y(t)), \quad t \in(0,1) \\
x(0)=y(0)=0 \\
x(1)=\alpha[y] \\
y(1)=\beta[x]
\end{array}\right.
$$


where $f_{1}, f_{2}:(0,1) \times[0,+\infty)^{2} \rightarrow[0,+\infty)$ are continuous and may be singular at $t=0,1$, and $\alpha[x], \beta[x]$ are bounded linear functionals on $C[0,1]$ given by

$$
\alpha[y]=\int_{0}^{1} y(t) d A(t), \quad \beta[x]=\int_{0}^{1} x(t) d B(t)
$$

involving Stieltjes integrals, and $A, B$ are functions of bounded variation with positive measures. Note that (2) can be reformulated by

$$
\left\{\begin{array}{l}
x(t)=\int_{0}^{1} G_{1}(t, s) u(s) d s+\int_{0}^{1} H_{1}(t, s) v(s) d s, \\
y(t)=\int_{0}^{1} G_{2}(t, s) v(s) d s+\int_{0}^{1} H_{2}(t, s) u(s) d s,
\end{array}\right.
$$

where

$$
\begin{aligned}
& G_{1}(t, s)=\frac{\alpha[t] t}{\kappa} \int_{0}^{1} K(s, \tau) d B(\tau), H_{1}(t, s)=\frac{t}{\kappa} \int_{0}^{1} K(s, \tau) d A(\tau), \\
& G_{2}(t, s)=\frac{\beta[t] t}{\kappa} \int_{0}^{1} K(s, \tau) d A(\tau), H_{2}(t, s)=\frac{t}{\kappa} \int_{0}^{1} K(s, \tau) d B(\tau), \\
& K(t, s)= \begin{cases}t(1-s), & 0 \leq t \leq s \leq 1, \\
s(1-t), & 0 \leq s \leq t \leq 1 .\end{cases}
\end{aligned}
$$

In [23], the authors study the existence and multiplicity of positive solutions for the system of nonlinear Hammerstein integral equations

$$
\begin{aligned}
& u(x)=\int_{0}^{1} k_{1}(x, y) f_{1}(y, u(y), v(y), w(y)) d y, \\
& v(x)=\int_{0}^{1} k_{2}(x, y) f_{2}(y, u(y), v(y), w(y)) d y, \\
& w(x)=\int_{0}^{1} k_{2}(x, y) f_{3}(y, u(y), v(y), w(y)) d y,
\end{aligned}
$$

where $k_{i} \in C\left([0,1] \times[0,1], \mathbb{R}_{+}\right)$and $f_{i} \in C\left([0,1] \times \mathbb{R}_{+}^{3}, \mathbb{R}_{+}\right)$, for $i=1,2,3$. The authors use concave functions to characterize the growth and the behaviors of nonlinearities $f_{1}, f_{2}, f_{3}$, considering three cases: assuming firstly that all are superlinear; secondly, with all sublinear and the last case with two superlinear ones and the other one sublinear. Based on a priori estimates obtained by Jensen's integral inequality for concave functions, the authors use the fixed point index theory to establish the main result.

Recently, in [18], Infante and Minhós, extending the results on [20], to prove the existence, multiplicity, non-existence and localization results for nontrivial solutions of the system

$$
\left\{\begin{array}{l}
u(t)=\int_{0}^{1} k_{1}(t, s) g_{1}(s) f_{1}\left(s, u(s), u^{\prime}(s), v(s), v^{\prime}(s)\right) d s \\
v(t)=\int_{0}^{1} k_{2}(t, s) g_{2}(s) f_{2}\left(s, u(s), u^{\prime}(s), v(s), v^{\prime}(s)\right) d s .
\end{array}\right.
$$

To obtain their results, it is assumed some adequate assumptions in order to apply a fixed index theorem and cone theory. 
Motivated by this work we consider the coupled integral system (1). Our results are based on [20] and [15], extending the results to systems of coupled Hammerstein-type integral equations with nonlinearities in both unknown functions and their first derivatives.

To the best of our knowledge, it is the first time where coupled systems contain integral equations with nonlinearities depending on several derivatives of both variables and, moreover, the derivatives can be of different order on each variable and each equation. That is, both equations and both variables can have a different regularity. This detail is very important as it allows the application of our results, for example, to boundary value problems with coupled systems composed of differential equations of different orders and distinct boundary conditions on each unknown function. This issue will open new fields of applications to phenomena modeled by coupled systems requiring different types of regularity on each variables.

The arguments in this paper apply Guo-Krasnosel'skii compression-expansion theory on cones. Moreover, the kernel functions and the corresponding derivatives associated to the integral equations are nonnegative and verify some adequate sign and growth assumptions. The dependence of the derivatives is overcome by the construction of suitable cones taking into account certain conditions of sublinearity/superlinearity at the origin and at $+\infty$.

The paper is organized as it follows: Sect. 2 contains the functional backgrounds. In Sect. 3, we present an existence result. Finally, an example shows the type of applications for these integral systems and boundary value problems allowed by our result.

\section{Backgrounds and assumptions}

In this paper we consider the cones defined, for $\iota=1,2$, by

$$
K_{\iota}:=\left\{w \in C^{r_{\iota}}[0,1]: \min _{t \in\left[a_{i l}, b_{i l}\right]} w^{(i)}(t) \geq c_{i l}\left\|w^{(i)}\right\|_{C^{r}}, \text { for } i=0,1, \ldots, r_{\iota}\right\}
$$

where $0<c_{i l}<1$ and $r_{\iota}=\max \left\{m_{\iota}, n_{\iota}\right\}$. The Banach space $C^{k}[0,1]$, equipped with the norm $\|\cdot\|_{C^{k}}$, defined by

$$
\|w\|_{C^{k}}:=\max \left\{\left\|w^{(j)}\right\|: j=0,1, \ldots, k\right\}
$$

and $\|y\|:=\max _{t \in[0,1]}|y(t)|$.

Moreover, the set $E:=K_{1} \times K_{2}$ with the norm

$$
\left\|\left(u_{1}, u_{2}\right)\right\|_{E}:=\max \left\{\left\|u_{1}\right\|_{C^{r_{1}}},\left\|u_{2}\right\|_{C^{r_{2}}}\right\}
$$

is a Banach space.

Definition 1 A function $h:[0,1] \times \mathbb{R}^{q} \rightarrow[0, \infty)$, for $q$ a positive integer, is $L^{\infty}$ Carathéodory if

(i) $h(\cdot, y)$ is measurable for each fixed $y \in \mathbb{R}^{q}$;

(ii) $h(t, \cdot)$ is continuous for a.e. $t \in[0,1]$;

(iii) for each $\rho>0$, there exists a function $\varphi_{\rho} \in L^{\infty}([0,1])$ such that $h(t, y) \leq \varphi_{\rho}(t)$ for $y \in[-\rho, \rho]$ and a.e. $t \in[0,1]$. 
The existence tool will be the well-known Guo-Krasnosel'skii results in expansive and compressive cones theory:

Lemma $2([16])$ Let $(E,\|\cdot\|)$ be a Banach space, and $P \subset E$ be a cone in E. Assume that $\Omega_{1}$ and $\Omega_{2}$ are open subsets of $E$ such that $0 \in \Omega_{1}, \overline{\Omega_{1}} \subset \Omega_{2}$.

If $T: P \cap\left(\overline{\Omega_{2}} \backslash \Omega_{1}\right) \rightarrow P$ is a completely continuous operator such that either

(i) $\|T u\| \leq\|u\|, u \in P \cap \partial \Omega_{1}$, and $\|T u\| \geq\|u\|, u \in P \cap \partial \Omega_{2}$, or

(ii) $\|T u\| \geq\|u\|, u \in P \cap \partial \Omega_{1}$, and $\|T u\| \leq\|u\|, u \in P \cap \partial \Omega_{2}$, then $T$ has a fixed point in $P \cap\left(\overline{\Omega_{2}} \backslash \Omega_{1}\right)$.

In this paper we will assume the following conditions.

(A1) For $\iota=1,2$, the function $k_{\iota}:[0,1]^{2} \rightarrow \mathbb{R}, k_{\iota} \in W^{r_{\iota}, 1}\left([0,1]^{2}\right)$, verify for all $\tau \in[0,1]$,

$$
\lim _{t \rightarrow \tau}\left|k_{\iota}(t, s)-k_{\iota}(\tau, s)\right|=0, \quad \text { for a.e. } s \in[0,1]
$$

and

$$
\lim _{t \rightarrow \tau}\left|\frac{\partial^{i} k_{l}}{\partial t^{i}}(t, s)-\frac{\partial^{i} k_{l}}{\partial t^{i}}(\tau, s)\right|=0, \quad \text { for a.e. } s \in[0,1] \text { and } i=1, \ldots, r_{\iota} .
$$

(A2) For $\iota=1,2$, and every $j_{\iota}=0,1, \ldots, r_{\iota}$, there exist subintervals $\left[a_{\imath}, b_{l j}\right] \subseteq[0,1]$, positive functions $\phi_{l j} \in L^{\infty}[0,1]$, and constants $c_{l j} \in(0,1]$, such that

$$
\begin{aligned}
& 0 \leq k_{\iota}(t, s) \leq \phi_{l 0}(s) \quad \text { for } t \in[0,1] \text { and a.e. } s \in[0,1] \\
& 0 \leq \frac{\partial^{i} k_{\iota}}{\partial t^{i}}(t, s) \leq \phi_{l i}(s) \quad \text { for } t \in[0,1], \text { a.e. } s \in[0,1] \text { and } i=1, \ldots, r_{\iota} \\
& k_{\iota}(t, s) \geq c_{\iota 0} \phi_{\iota 0}(s) \quad \text { for } t \in\left[a_{\iota 0}, b_{\iota 0}\right] \text { and a.e. } s \in[0,1] \\
& \frac{\partial^{i} k_{\iota}}{\partial t^{i}}(t, s) \geq c_{l i} \phi_{l i}(s) \quad \text { for } t \in\left[a_{\iota i}, b_{l i}\right] \text {, a.e. } s \in[0,1] \text { and } i=1, \ldots, r_{\iota} .
\end{aligned}
$$

(A3) For $\iota=1,2, j_{\iota}=0,1, \ldots, r_{\iota}, g_{\iota} \in L^{1}([0,1]), g_{\iota}(t) \geq 0$ a.e. $t \in[0,1], \phi_{l j} \in L^{\infty}[0,1]$ and $\int_{a_{l j}}^{b_{l j}} \phi_{l j}(s) g_{\iota}(s) d s>0$.

Consider the following growth assumptions:

(B1) For $\iota=1,2, l=0,1, \ldots, m_{\iota}, j=0,1, \ldots, n_{\iota}$,

$$
\limsup _{x_{l} \rightarrow 0, y_{j} \rightarrow 0} \max _{t \in[0,1]} \frac{f_{\iota}\left(t, x_{0}, \ldots, x_{m_{l}}, y_{0}, \ldots, y_{n_{\iota}}\right)}{\max \left\{\left|x_{l}\right|,\left|y_{j}\right|\right\}}=0
$$

and

$$
\liminf _{x_{l} \rightarrow+\infty, y_{j} \rightarrow+\infty} \min _{t \in[0,1]} \frac{f_{l}\left(t, x_{0}, \ldots, x_{m_{l}}, y_{0}, \ldots, y_{n_{l}}\right)}{\max \left\{\left|x_{l}\right|,\left|y_{j}\right|\right\}}=+\infty
$$

(B2) For $\iota=1,2, l=0,1, \ldots, m_{\iota}, j=0,1, \ldots, n_{\iota}$,

$$
\liminf _{x_{l} \rightarrow 0, y_{j} \rightarrow 0} \min _{t \in[0,1]} \frac{f_{\iota}\left(t, x_{0}, \ldots, x_{m_{l}}, y_{0}, \ldots, y_{n_{t}}\right)}{\max \left\{\left|x_{l}\right|,\left|y_{j}\right|\right\}}=+\infty
$$


and

$$
\limsup _{x_{l} \rightarrow+\infty, y_{j} \rightarrow+\infty} \max _{t \in[0,1]} \frac{f_{l}\left(t, x_{0}, x_{1}, \ldots, x_{m_{l}+n_{l}+1}\right)}{\max \left\{\left|x_{l}\right|,\left|y_{j}\right|\right\}}=0 .
$$

\section{Main result}

The main result is given by next theorem:

Theorem 3 Let, for $\iota=1,2, f_{\iota}:[0,1] \times \mathbb{R}^{m_{\iota}+n_{\iota}+2} \rightarrow[0, \infty)$ be $L^{\infty}$-Carathéodory functions such that assumptions (A1)-(A3) hold and or conditions (B1), or (B2), are satisfied. Then problem (1) has at least one positive solution $(u, v) \in\left(C^{r_{1}}[0,1] \times C^{r_{2}}[0,1]\right)$.

Proof Consider the cones $K_{\iota}$ in (3), the Banach space $E:=K_{1} \times K_{2}$ with the norm given by (4), and define the operators $T_{1}: E \rightarrow K_{1}$ and $T_{2}: E \rightarrow K_{2}$ such that

$$
\left\{\begin{array}{l}
T_{1}\left(u_{1}, u_{2}\right)(t)=\int_{0}^{1} k_{1}(t, s) g_{1}(s) f_{1}\left(s, u_{1}(s), \ldots, u_{1}^{\left(m_{1}\right)}(s), u_{2}(s), \ldots, u_{2}^{\left(n_{1}\right)}(s)\right) d s \\
T_{2}\left(u_{1}, u_{2}\right)(t)=\int_{0}^{1} k_{2}(t, s) g_{2}(s) f_{2}\left(s, u_{1}(s), \ldots, u_{1}^{\left(m_{2}\right)}(s), u_{2}(s), \ldots, u_{2}^{\left(n_{2}\right)}(s)\right) d s .
\end{array}\right.
$$

The proof will be done in several steps and the main idea is to show that the operator $T: E \rightarrow E$ defined by $T=\left(T_{1}, T_{2}\right)$ has a fixed point on $E$. For this, according to Lemma 2, we need to show that $T$ is completely continuous.

Step 1: $T: E \rightarrow E$ is well defined in $E$.

It will be enough to prove that $T_{\iota}$ are well defined in $K_{\iota}$, for $\iota=1,2$.

Take $\left(u_{1}, u_{2}\right) \in E$. By $(\mathrm{A} 2)$,

$$
\begin{aligned}
\left\|T_{1}\left(u_{1}, u_{2}\right)\right\| & =\max _{t \in[0,1]} \int_{0}^{1} k_{1}(t, s) g_{1}(s) f_{1}\left(s, u_{1}(s), \ldots, u_{1}^{\left(m_{1}\right)}(s), u_{2}(s), \ldots, u_{2}^{\left(n_{1}\right)}(s)\right) d s \\
& \leq \int_{0}^{1} \phi_{10}(s) g_{1}(s) f_{1}\left(s, u_{1}(s), \ldots, u_{1}^{\left(m_{1}\right)}(s), u_{2}(s), \ldots, u_{2}^{\left(n_{1}\right)}(s)\right) d s
\end{aligned}
$$

and

$$
\begin{aligned}
\min _{t \in\left[a_{10}, b_{10}\right]} T_{1}\left(u_{1}, u_{2}\right)(t) & \geq c_{10} \int_{0}^{1} \phi_{10}(s) g_{1}(s) f_{1}\left(s, u_{1}(s), \ldots, u_{1}^{\left(m_{1}\right)}(s), u_{2}(s), \ldots, u_{2}^{\left(n_{1}\right)}(s)\right) d s \\
& \geq c_{10}\left\|T_{1}\left(u_{1}, u_{2}\right)\right\| .
\end{aligned}
$$

On the other hand, for $i=1, \ldots, r_{1}$,

$$
\begin{aligned}
\| & \left(T_{1}\left(u_{1}, u_{2}\right)\right)^{(i)} \| \\
\quad & \max _{t \in[0,1]}\left|\int_{0}^{1} \frac{\partial^{i} k_{1}}{\partial t^{i}}(t, s) g_{1}(s) f_{1}\left(s, u_{1}(s), \ldots, u_{1}^{\left(m_{1}\right)}(s), u_{2}(s), \ldots, u_{2}^{\left(n_{1}\right)}(s)\right) d s\right| \\
& \leq \int_{0}^{1} \phi_{1 i}(s) g_{1}(s) f_{1}\left(s, u_{1}(s), \ldots, u_{1}^{\left(m_{1}\right)}(s), u_{2}(s), \ldots, u_{2}^{\left(n_{1}\right)}(s)\right) d s
\end{aligned}
$$

and

$$
\begin{aligned}
& \min _{t \in\left[a_{1 i}, b_{1 i}\right]}\left(T_{1}\left(u_{1}, u_{2}\right)\right)^{(i)} \\
& =\min _{t \in\left[a_{1 i}, b_{1 i}\right]} \int_{0}^{1} \frac{\partial^{i} k_{1}}{\partial t^{i}}(t, s) g_{1}(s) f_{1}\left(s, u_{1}(s), \ldots, u_{1}^{\left(m_{1}\right)}(s), u_{2}(s), \ldots, u_{2}^{\left(n_{1}\right)}(s)\right) d s
\end{aligned}
$$




$$
\begin{aligned}
& \geq c_{1 i} \int_{0}^{1} \phi_{1 i}(s) g_{1}(s) f_{1}\left(s, u_{1}(s), \ldots, u_{1}^{\left(m_{1}\right)}(s), u_{2}(s), \ldots, u_{2}^{\left(n_{1}\right)}(s)\right) d s \\
& \geq c_{1 i}\left\|\left(T_{1}\left(u_{1}, u_{2}\right)\right)^{(i)}\right\| .
\end{aligned}
$$

So, for $i=0,1, \ldots, r_{1}$,

$$
\min _{t \in\left[a_{1 i}, b_{1 i}\right]} T_{1}\left(u_{1}, u_{2}\right)(t) \geq d_{1}\left\|T_{1}\left(u_{1}, u_{2}\right)\right\|_{C^{r_{1}}}
$$

with $0<d_{1} \leq \max \left\{c_{1 i}, i=0,1, \ldots, r_{1}\right\} \leq 1$.

Therefore, $T_{1} E \subseteq K_{1}$. The inclusion $T_{2} E \subseteq K_{2}$ can be proved similarly, and consequently $T E \subset E$.

\section{Step 2: $T$ is uniformly bounded in $E$.}

We will prove that $T_{1}$ and $T_{2}$ are uniformly bounded in $K_{1}$ and $K_{2}$, respectively.

Consider $\left(u_{1}, u_{2}\right) \in E$ such that $\left\|\left(u_{1}, u_{2}\right)\right\|_{E} \leq \rho$, for some $\rho>0$.

The proof will be done for the operator $T_{1}$, as for $T_{2}$ the arguments are analogous.

By (A2), (A3) and Definition 1,

$$
\begin{aligned}
\left\|T_{1}\left(u_{1}, u_{2}\right)\right\| & =\max _{t \in[0,1]}\left|T_{1}\left(u_{1}, u_{2}\right)(t)\right| \\
& \leq \int_{0}^{1} \phi_{10}(s) g_{1}(s) f_{1}\left(s, u_{1}(s), \ldots, u_{1}^{\left(m_{1}\right)}(s), u_{2}(s), \ldots, u_{2}^{\left(n_{1}\right)}(s)\right) d s \\
& \leq \int_{0}^{1} \phi_{10}(s) g_{1}(s) \varphi_{\rho}(s) d s<+\infty,
\end{aligned}
$$

and, for $i=1, \ldots, r_{1}$,

$$
\begin{aligned}
\| & \left(T_{1}\left(u_{1}, u_{2}\right)\right)^{(i)} \| \\
& =\max _{t \in[0,1]}\left|\int_{0}^{1} \frac{\partial^{(i)} k_{1}}{\partial t^{i}}(t, s) g_{1}(s) f_{1}\left(s, u_{1}(s), \ldots, u_{1}^{\left(m_{1}\right)}(s), u_{2}(s), \ldots, u_{2}^{\left(n_{1}\right)}(s)\right) d s\right| \\
& \leq \int_{0}^{1} \phi_{1 i}(s) g_{1}(s) f_{1}\left(s, u_{1}(s), \ldots, u_{1}^{\left(m_{1}\right)}(s), u_{2}(s), \ldots, u_{2}^{\left(n_{1}\right)}(s)\right) d s \\
& \leq \int_{0}^{1} \phi_{1 i}(s) g_{1}(s) \varphi_{\rho}(s) d s<+\infty .
\end{aligned}
$$

Therefore $\left\|T_{1}\left(u_{1}, u_{2}\right)\right\|_{C^{r_{1}}}<+\infty$, and, so, $T_{1}$ is uniformly bounded in $K_{1}$.

By an analogous method it can de proved that $T_{2}$ is uniformly bounded in $K_{2}$, and, therefore, $T$ is uniformly bounded in $E$.

Step 3: $T$ is equicontinuous in $E$.

This step will be shown if $T_{1}$ and $T_{2}$ are equicontinuous in $K_{1}$ and $K_{2}$, respectively. The calculus will be done only for $T_{1}$, as the other case is similar.

Consider $t_{1}, t_{2} \in[0,1]$. By (A1),

$$
\begin{aligned}
& \left|T_{1}\left(u_{1}, u_{2}\right)\left(t_{1}\right)-T_{1}\left(u_{1}, u_{2}\right)\left(t_{2}\right)\right| \\
& \quad \leq \int_{0}^{1}\left|k_{1}\left(t_{1}, s\right)-k_{1}\left(t_{2}, s\right)\right| g_{1}(s) f_{1}\left(s, u_{1}(s), \ldots, u_{1}^{\left(m_{1}\right)}(s), u_{2}(s), \ldots, u_{2}^{\left(n_{1}\right)}(s)\right) d s
\end{aligned}
$$




$$
\leq \int_{0}^{1}\left|k_{1}\left(t_{1}, s\right)-k_{1}\left(t_{2}, s\right)\right| g_{1}(s) \varphi_{\rho}(s) d s \rightarrow 0 \quad \text { as } t_{1} \rightarrow t_{2},
$$

and, for $i=1, \ldots, r_{1}$,

$$
\begin{aligned}
& \left|\left(T_{1}\left(u_{1}, u_{2}\right)\right)^{(i)}\left(t_{1}\right)-\left(T_{1}\left(u_{1}, u_{2}\right)\right)^{(i)}\left(t_{2}\right)\right| \\
& \quad \leq \int_{0}^{1}\left|\frac{\partial^{i} k_{1}}{\partial t^{i}}\left(t_{1}, s\right)-\frac{\partial^{i} k_{1}}{\partial t^{i}}\left(t_{2}, s\right)\right| g_{1}(s) f_{1}\left(s, u_{1}(s), \ldots, u_{1}^{\left(m_{1}\right)}(s), u_{2}(s), \ldots, u_{2}^{\left(n_{1}\right)}(s)\right) d s \\
& \quad \leq \int_{0}^{1}\left|\frac{\partial^{i} k_{1}}{\partial t^{i}}\left(t_{1}, s\right)-\frac{\partial^{i} k_{1}}{\partial t^{i}}\left(t_{2}, s\right)\right| g_{1}(s) \varphi_{\rho}(s) d s \rightarrow 0 \quad \text { as } t_{1} \rightarrow t_{2} .
\end{aligned}
$$

Therefore, $T_{1}$ is equicontinuous in $K_{1}$.

In the same way it can be proved that $T_{2}$ is equicontinuous in $K_{2}$. Then $T$ is equicontinuous in $E$.

By the Arzelà-Ascoli theorem, $T$ is completely continuous in $E$.

Assume that condition (B1) holds.

Step 4: $\left\|T\left(u_{1}, u_{2}\right)\right\|_{E} \leq\left\|\left(u_{1}, u_{2}\right)\right\|_{E}$, for $\left(u_{1}, u_{2}\right) \in E \cap \partial \Omega_{1}$ with $\Omega_{1}=\left\{\left(u_{1}, u_{2}\right) \in E\right.$ : $\left.\left\|\left(u_{1}, u_{2}\right)\right\|_{E}<\rho_{1}\right\}$, for some $\rho_{1}>0$.

To prove that

$$
\max \left\{\left\|T_{1}\left(u_{1}, u_{2}\right)\right\|_{C^{r_{1}}},\left\|T_{2}\left(u_{1}, u_{2}\right)\right\|_{C^{r_{2}}}\right\} \leq\left\|\left(u_{1}, u_{2}\right)\right\|_{E^{\prime}}
$$

it will be enough to show that

$$
\left\|T_{1}\left(u_{1}, u_{2}\right)\right\|_{C^{r_{1}}} \leq\left\|\left(u_{1}, u_{2}\right)\right\|_{E} \quad \text { and } \quad\left\|T_{2}\left(u_{1}, u_{2}\right)\right\|_{C^{r_{2}}} \leq\left\|\left(u_{1}, u_{2}\right)\right\|_{E} \text {. }
$$

As $\left(u_{1}, u_{2}\right) \in E \cap \partial \Omega_{1}$ then $\left\|\left(u_{1}, u_{2}\right)\right\|_{E}=\rho_{1}$.

For $i=0,1, \ldots, r_{1}$, and (A3), let us define

$$
\varepsilon_{1}:=\min \left\{\frac{1}{\int_{0}^{1} \phi_{1 i}(s) g_{1}(s) d s}\right\}
$$

By (5), there exists $0<\rho_{1}<1$ such that

$$
f_{1}\left(t, u_{1}(t), \ldots, u_{1}^{\left(m_{1}\right)}(t), u_{2}(t), \ldots, u_{2}^{\left(n_{1}\right)}(t)\right) \leq \varepsilon_{1}\left\|\left(u_{1}, u_{2}\right)\right\|_{E^{\prime}}
$$

for $\left\|\left(u_{1}, u_{2}\right)\right\|_{E} \leq \rho_{1}$.

By (A2), (11), and (10),

$$
\begin{aligned}
T_{1}\left(u_{1}, u_{2}\right)(t) & =\int_{0}^{1} k_{1}(t, s) g_{1}(s) f_{1}\left(s, u_{1}(s), \ldots, u_{1}^{\left(m_{1}\right)}(s), u_{2}(s), \ldots, u_{2}^{\left(n_{1}\right)}(s)\right) d s \\
& \leq \int_{0}^{1} \phi_{10}(s) g_{1}(s) \varepsilon_{1}\left\|\left(u_{1}, u_{2}\right)\right\|_{E} d s \\
& =\varepsilon_{1} \rho_{1} \int_{0}^{1} \phi_{10}(s) g_{1}(s) d s<\rho_{1}=\left\|\left(u_{1}, u_{2}\right)\right\|_{E},
\end{aligned}
$$


and, for $i=1, \ldots, r_{1}$,

$$
\begin{aligned}
\left(T_{1}\left(u_{1}, u_{2}\right)(t)\right)^{(i)} & =\int_{0}^{1} \frac{\partial^{i} k_{l}}{\partial t^{i}}(t, s) g_{1}(s) f_{\iota}\left(s, u_{1}(s), \ldots, u_{1}^{\left(m_{1}\right)}(s), u_{2}(s), \ldots, u_{2}^{\left(n_{1}\right)}(s)\right) d s \\
& \leq \int_{0}^{1} \phi_{1 i}(s) g_{1}(s) \varepsilon_{1}\left\|\left(u_{1}, u_{2}\right)\right\|_{E} d s<\rho_{1}=\left\|\left(u_{1}, u_{2}\right)\right\|_{E} .
\end{aligned}
$$

Ergo, $\left\|T_{1}\left(u_{1}, u_{2}\right)\right\|_{C^{r_{1}}} \leq\left\|\left(u_{1}, u_{2}\right)\right\|_{E}$, for $\left(u_{1}, u_{2}\right) \in E \cap \partial \Omega_{1}$. By similar calculations it can be proved that $\left\|T_{2}\left(u_{1}, u_{2}\right)\right\|_{C^{r_{2}}} \leq\left\|\left(u_{1}, u_{2}\right)\right\|_{E}$ and, therefore, $\left\|T\left(u_{1}, u_{2}\right)\right\|_{E} \leq\left\|\left(u_{1}, u_{2}\right)\right\|_{E}$, for $\left(u_{1}, u_{2}\right) \in E \cap \partial \Omega_{1}$.

Step 5: $\left\|T\left(u_{1}, u_{2}\right)\right\|_{E} \geq\left\|\left(u_{1}, u_{2}\right)\right\|_{E}$, for $u \in E \cap \partial \Omega_{2}$ with $\Omega_{2}=\left\{\left(u_{1}, u_{2}\right) \in E:\left\|\left(u_{1}, u_{2}\right)\right\|_{E}<\right.$ $\left.\rho_{2}\right\}$, for some $\rho_{2}>0$.

If there exists $i_{0} \in\left\{0,1, \ldots, m_{1}\right\}$, or $j_{0} \in\left\{0,1, \ldots, n_{1}\right\}$, such that $u_{1}^{\left(i_{0}\right)}(t) \rightarrow+\infty$ and $u_{2}^{\left(j_{0}\right)}(t) \rightarrow+\infty$, then $\left\|\left(u_{1}, u_{2}\right)\right\|_{E} \rightarrow+\infty$.

By (6), for $\iota=1,2$, there exist $\rho_{\iota}^{*}>0$ and $\theta>0$, such that, when $\left\|\left(u_{1}, u_{2}\right)\right\|_{E} \geq \theta$, we have

$$
f_{1}\left(t, u_{1}(t), \ldots, u_{1}^{\left(m_{1}\right)}(t), u_{2}(t), \ldots, u_{2}^{\left(n_{1}\right)}(t)\right) \geq\left\|\left(u_{1}, u_{2}\right)\right\|_{E} .
$$

Define, for $i=0,1, \ldots, r_{1}$,

$$
\xi_{1}:=\max \left\{\frac{1}{c_{1 i} \int_{0}^{1} \phi_{1 i}(s) g_{1}(s) d s}\right\} .
$$

Let $\left(u_{1}, u_{2}\right) \in E$ be such that $\left\|\left(u_{1}, u_{2}\right)\right\|_{E}=\rho_{2}$, with $\rho_{2}>\rho_{1}$.

Now from (A2), and (13),

$$
\begin{aligned}
T_{1}\left(u_{1}, u_{2}\right)(t) & \geq \int_{a_{10}}^{b_{10}} k_{1}(t, s) g_{1}(s) f_{1}\left(s, u_{1}(s), \ldots, u_{1}^{\left(m_{1}\right)}(s), u_{2}(s), \ldots, u_{2}^{\left(n_{1}\right)}(s)\right) d s \\
& \geq c_{10} \int_{a_{10}}^{b_{10}} \phi_{10}(s) g_{1}(s) f_{1}\left(s, u_{1}(s), \ldots, u_{1}^{\left(m_{1}\right)}(s), u_{2}(s), \ldots, u_{2}^{\left(n_{1}\right)}(s)\right) d s \\
& \geq c_{10} \int_{a_{10}}^{b_{10}} \phi_{10}(s) g_{1}(s) \xi_{1}\left\|\left(u_{1}, u_{2}\right)\right\|_{E} d s \\
& =c_{10} \xi_{1} \rho_{2} \int_{a_{10}}^{b_{10}} \phi_{10}(s) g_{1}(s) d s \geq \rho_{2}=\left\|\left(u_{1}, u_{2}\right)\right\|_{E},
\end{aligned}
$$

and analogously, for $i=1, \ldots, r_{1}$,

$$
\begin{aligned}
\left(T_{1}\left(u_{1}, u_{2}\right)(t)\right)^{(i)} & \geq \int_{a_{1 i}}^{b_{1 i}} \frac{\partial^{(i)} k_{1}}{\partial t^{i}}(t, s) g_{1}(s) f_{1}\left(s, u_{1}(s), \ldots, u_{1}^{\left(m_{1}\right)}(s), u_{2}(s), \ldots, u_{2}^{\left(n_{1}\right)}(s)\right) d s \\
& \geq c_{1 i} \int_{a_{1 i}}^{b_{1 i}} \phi_{1 i}(s) g_{1}(s) f_{1}\left(s, u_{1}(s), \ldots, u_{1}^{\left(m_{1}\right)}(s), u_{2}(s), \ldots, u_{2}^{\left(n_{1}\right)}(s)\right) d s \\
& \geq c_{1 i} \xi_{1} \rho_{2} \int_{a_{1 i}}^{b_{1 i}} \phi_{1 i}(s) g_{1}(s) d s \geq \rho_{2}=\left\|\left(u_{1}, u_{2}\right)\right\|_{E} .
\end{aligned}
$$

Therefore, $\left\|T_{1}\left(u_{1}, u_{2}\right)\right\|_{C^{r_{1}}} \geq\left\|\left(u_{1}, u_{2}\right)\right\|_{E}$, for $\left(u_{1}, u_{2}\right) \in E \cap \partial \Omega_{2}$. Analogously it can be shown that $\left\|T_{2}\left(u_{1}, u_{2}\right)\right\|_{C^{r_{2}}} \geq\left\|\left(u_{1}, u_{2}\right)\right\|_{E}$, for $\left(u_{1}, u_{2}\right) \in E \cap \partial \Omega_{2}$, and, therefore, $\left\|T\left(u_{1}, u_{2}\right)\right\|_{E} \geq\left\|\left(u_{1}, u_{2}\right)\right\|_{E}$, for $\left(u_{1}, u_{2}\right) \in E \cap \partial \Omega_{2}$. 
By Lemma 2, the operator $T$ has a fixed point in $K \cap\left(\overline{\Omega_{2}} \backslash \Omega_{1}\right)$ which in turn is a solution of our problem.

Now assume that (B2) holds.

Step 6: $\left\|T\left(u_{1}, u_{2}\right)\right\|_{E} \geq\left\|\left(u_{1}, u_{2}\right)\right\|_{E}$, for $u \in E \cap \partial \Omega_{3}$ with $\Omega_{3}=\left\{\left(u_{1}, u_{2}\right) \in E:\left\|\left(u_{1}, u_{2}\right)\right\|_{E}<\right.$ $\left.\rho_{3}\right\}$, for some $\rho_{3}>0$.

Taking $\xi_{1}>0$ as in (13), we see that by (7) there exists $0<\rho_{3}<1$ such that

$$
\left(t, u_{1}(t), \ldots, u_{1}^{\left(m_{1}\right)}(t), u_{2}(t), \ldots, u_{2}^{\left(n_{1}\right)}(t)\right) \in[0,1] \times\left[0, \rho_{3_{l}}\right]^{m_{1}+n_{1}+2}
$$

and

$$
f_{1}\left(t, u_{1}(t), \ldots, u_{1}^{\left(m_{1}\right)}(t), u_{2}(t), \ldots, u_{2}^{\left(n_{1}\right)}(t)\right) \geq \xi_{\iota}\left\|\left(u_{1}, u_{2}\right)\right\|_{E}
$$

Consider $\left(u_{1}, u_{2}\right) \in E$ such that $\left\|\left(u_{1}, u_{2}\right)\right\|_{E}=\rho_{3_{l}}$. Then, applying similar inequalities to Step 5 , we obtain $\left\|T\left(u_{1}, u_{2}\right)\right\|_{E} \geq\left\|\left(u_{1}, u_{2}\right)\right\|_{E}$.

Step 7: $\left\|T\left(u_{1}, u_{2}\right)\right\|_{E} \leq\left\|\left(u_{1}, u_{2}\right)\right\|_{E}$, for $\left(u_{1}, u_{2}\right) \in E \cap \partial \Omega_{4}$ with $\Omega_{4}=\left\{\left(u_{1}, u_{2}\right) \in E\right.$ : $\left.\left\|\left(u_{1}, u_{2}\right)\right\|_{E}<\rho_{4}\right\}$, for some $\rho_{4}>0$.

Case 7.1. Suppose that $f_{1}$ is bounded.

Then there is an $N>0$ such that $f_{1}\left(t, u_{1}(t), \ldots, u_{1}^{\left(m_{1}\right)}(t), u_{2}(t), \ldots, u_{2}^{\left(n_{1}\right)}(t)\right) \leq N$ for all $\left(t, u_{1}(t), \ldots, u_{1}^{\left(m_{1}\right)}(t), u_{2}(t), \ldots, u_{2}^{\left(n_{1}\right)}(t)\right) \in[0,1] \times[0,+\infty)^{m_{1}+n_{1}+2}$. Choose

$$
\rho_{4}:=\max \left\{\rho_{3}+1, N \int_{0}^{1} \phi_{1 i}(s) g_{1}(s) d s: i=0,1, \ldots, r_{1}\right\}
$$

and take $\left(u_{1}, u_{2}\right) \in E$ with $\left\|\left(u_{1}, u_{2}\right)\right\|_{E}=\rho_{4}$. Then

$$
\begin{aligned}
T_{1}\left(u_{1}, u_{2}\right)(t) & =\int_{0}^{1} k_{1}(t, s) g_{1}(s) f_{1}\left(s, u_{1}(s), \ldots, u_{1}^{\left(m_{1}\right)}(s), u_{2}(s), \ldots, u_{2}^{\left(n_{1}\right)}(s)\right) d s \\
& \leq N \int_{0}^{1} \phi_{10}(s) g_{1}(s) d s \leq \rho_{4}, \quad \text { for } t \in[0,1],
\end{aligned}
$$

and for $i=1, \ldots, r_{1}$,

$$
\begin{aligned}
\left(T_{1}\left(u_{1}, u_{2}\right)(t)\right)^{(i)} & =\int_{0}^{1} \frac{\partial^{i} k_{1}}{\partial t^{i}}(t, s) g_{1}(s) f_{1}\left(s, u_{1}(s), \ldots, u_{1}^{\left(m_{1}\right)}(s), u_{2}(s), \ldots, u_{2}^{\left(n_{1}\right)}(s)\right) d s \\
& \leq N \int_{0}^{1} \phi_{1 i}(s) g_{1}(s) d s \leq \rho_{4}, \quad \text { for } t \in[0,1] .
\end{aligned}
$$

Thus, $\left\|T_{1}\left(u_{1}, u_{2}\right)\right\|_{C^{r_{1}}} \leq\left\|\left(u_{1}, u_{2}\right)\right\|_{E}$.

The same arguments can be applied to show that $\left\|T_{2}\left(u_{1}, u_{2}\right)\right\|_{C^{r_{2}}} \leq\left\|\left(u_{1}, u_{2}\right)\right\|_{E}$. So, $\left\|T\left(u_{1}, u_{2}\right)\right\|_{E} \leq\left\|\left(u_{1}, u_{2}\right)\right\|_{E}$.

Case 7.2. Suppose that $f_{1}$ is unbounded.

By (8), there exists $\mu>0$ such that

$$
\max \left\{\mu \int_{0}^{1} \phi_{1 i}(s) g_{1}(s) d s: i=0,1, \ldots, r_{1}\right\} \leq 1
$$


and

$$
f_{1}\left(t, u_{1}(t), \ldots, u_{1}^{\left(m_{1}\right)}(t), u_{2}(t), \ldots, u_{2}^{\left(n_{1}\right)}(t)\right) \leq \mu\left\|\left(u_{1}, u_{2}\right)\right\|_{E},
$$

for every $M>0$ such that $\left\|\left(u_{1}, u_{2}\right)\right\|_{E} \geq M$.

Define

$$
\rho_{4}:=\max \left\{M, \rho_{3}+1\right\}
$$

Then, for $\left(u_{1}, u_{2}\right) \in E \cap \partial \Omega_{4}$, we have $\left\|\left(u_{1}, u_{2}\right)\right\|_{E}=\rho_{4}$ and, by (16),

$$
f_{1}\left(t, u_{1}(t), \ldots, u_{1}^{\left(m_{1}\right)}(t), u_{2}(t), \ldots, u_{2}^{\left(n_{1}\right)}(t)\right) \leq \mu\left\|\left(u_{1}, u_{2}\right)\right\|_{E} \leq \mu \rho_{4} .
$$

So,

$$
T_{1}\left(u_{1}, u_{2}\right)(t) \leq \int_{0}^{1} \phi_{10}(s) g_{1}(s) \mu \rho_{4} d s \leq \mu \rho_{4} \int_{0}^{1} \phi_{10}(s) g_{1}(s) d s \leq \rho_{4},
$$

and for $i=1, \ldots, r_{1}$,

$$
\left(T_{1}\left(u_{1}, u_{2}\right)(t)\right)^{(i)} \leq \int_{0}^{1} \phi_{1 i}(s) g_{1}(s) \mu \rho_{4} d s \leq \mu \rho_{4} \int_{0}^{1} \phi_{1 i}(s) g_{1}(s) d s \leq \rho_{4} .
$$

Therefore, $\left\|T_{1}\left(u_{1}, u_{2}\right)\right\|_{C^{r_{1}}} \leq\left\|\left(u_{1}, u_{2}\right)\right\|_{E}$, for $\left(u_{1}, u_{2}\right) \in E \cap \partial \Omega_{4}$.

In the same way we can have $\left\|T_{2}\left(u_{1}, u_{2}\right)\right\|_{C^{r_{2}}} \leq\left\|\left(u_{1}, u_{2}\right)\right\|_{E}$, for $\left(u_{1}, u_{2}\right) \in E \cap \partial \Omega_{4}$, and, therefore, $\left\|T\left(u_{1}, u_{2}\right)\right\|_{E} \leq\left\|\left(u_{1}, u_{2}\right)\right\|_{E}$, for $\left(u_{1}, u_{2}\right) \in E \cap \partial \Omega_{4}$.

The remaining cases $\left(f_{2}\right.$ bounded or unbounded) can be processed by similar techniques.

By Lemma 2, the operator $T$ has a fixed point in $E \cap\left(\overline{\Omega_{4}} \backslash \Omega_{3}\right)$ that, in turn, is a solution of the problem.

\section{Example}

Consider the following coupled system composed of third and second nonlinear equations, with three-point boundary conditions:

$$
\left\{\begin{array}{l}
-u_{1}^{\prime \prime \prime}(t)=\left(t^{2}+1\right)\left(e^{-\left(u_{2}^{\prime}(t)+u_{1}(t)\right)^{2}}+\sqrt{\left|u_{1}^{\prime}(t)+u_{2}(t)\right|}\right) \\
u_{2}^{\prime \prime}(t)=t^{4}\left(2+\cos \left(u_{2}(t)+u_{1}(t)\right)^{2}\left(\sin \left(u_{1}^{\prime}(t) u_{2}^{\prime}(t)\right)+1\right),\right. \\
u(0)=u^{\prime}(0)=0, u^{\prime}(1)=\frac{3}{2} u^{\prime}\left(\frac{1}{2}\right) \\
v(0)=0, v^{\prime}(1)=\frac{3}{2} v^{\prime}\left(\frac{1}{2}\right)
\end{array}\right.
$$

Note that the problem (18) can be rewritten as the following system of integral equations:

$$
\left\{\begin{array}{l}
u_{1}(t)=\int_{0}^{1} k_{1}(t, s)\left(s^{2}+1\right)\left(e^{-\left(u_{2}^{\prime}(s)+u_{1}(s)\right)^{2}}+\sqrt{\left|u_{1}^{\prime}(s)+u_{2}(s)\right|}\right) d s, \\
u_{2}(t)=\int_{0}^{1} k_{2}(t, s) s^{4}\left(2+\cos \left(u_{2}(s)+u_{1}(s)\right)^{2}\left(\sin \left(u_{1}^{\prime}(s) u_{2}^{\prime}(s)\right)+1\right) d s,\right.
\end{array}\right.
$$


where the kernel functions $k_{1}(t, s)$ and $k_{2}(t, s)$ are given by the corresponding Green's functions

$$
\begin{aligned}
& G_{1}(t, s)= \begin{cases}t s-\frac{s^{2}}{2}+2 t^{2} s, & s \leq \min \left\{\frac{1}{2}, t\right\}, \\
\frac{t^{2}}{2}+2 t^{2} s, & t \leq s \leq \frac{1}{2}, \\
t s-\frac{s^{2}}{2}+t^{2}\left(\frac{3}{2}-2 s\right), & \frac{1}{2} \leq s \leq t, \\
2 t^{2}(1-s), & \max \left\{\frac{1}{2}, t\right\} \leq s,\end{cases} \\
& G_{2}(t, s)= \begin{cases}t-s, & s \leq t \leq 1, \\
2 t, & \frac{1}{2} \leq t \leq s \leq 1,\end{cases}
\end{aligned}
$$

respectively.

Clearly system (19) is a particular case of (1) with $r_{1}=r_{2}=m_{1}=m_{2}=n_{1}=n_{2}=1, g_{1}(t)=$ $t^{2}+1, g_{2}(t)=t^{4}, k_{1}(t, s)=G_{1}(t, s), k_{2}(t, s)=G_{2}(t, s)$, and

$$
\begin{aligned}
& f_{1}(t, x, y, z, w)=\left(e^{-(w+x)^{2}}+\sqrt{|y+z|}\right), \\
& f_{2}(t, x, y, z, w)=(2+\cos (z+x))^{2}(\sin (y w)+1) .
\end{aligned}
$$

These functions $f_{1}, f_{2}: \mathbb{R}^{5} \rightarrow[0, \infty)$ are $L^{\infty}$-Carathéodory as, for $\rho>0$, when $\max \{|x|,|y|$, $|z|,|w|\}<\rho$, there exist functions $\varphi_{1 \rho}, \varphi_{2 \rho} \in L^{\infty}([0,1])$ such that

$$
\begin{aligned}
& f_{1}(t, x, y, z, w) \leq(1+\sqrt{|2 \rho|}):=\varphi_{1 \rho} \\
& f_{2}(t, x, y, z, w)=(2+\cos (z+x))^{2}(\sin (y w)+1) \leq 18:=\varphi_{2} .
\end{aligned}
$$

The first derivative of the Green's functions are positive, with

$$
\begin{aligned}
& \frac{\partial G_{1}}{\partial t}(t, s)= \begin{cases}s+4 t s, & s \leq \min \left\{\frac{1}{2}, t\right\} \\
t+4 t s, & t \leq s \leq \frac{1}{2}, \\
t+2 t\left(\frac{3}{2}-2 s\right), & \frac{1}{2} \leq s \leq t, \\
4 t(1-s), & \max \left\{\frac{1}{2}, t\right\} \leq s,\end{cases} \\
& \frac{\partial G_{2}}{\partial t}(t, s)= \begin{cases}1, & s \leq t \leq 1, \\
2, & \frac{1}{2} \leq t \leq s \leq 1\end{cases}
\end{aligned}
$$

Therefore (A1) holds and, to show that (A2) is verified, we follow the arguments in [15] (Lemmas 4.1-4.4), for $G_{1}$ and any $(t, s) \in[0,1] \times[0,1]$, to obtain

$$
0 \leq G_{1}(t, s) \leq 10 s(1-s):=\phi_{10}(s), \quad 0 \leq \frac{\partial G_{1}}{\partial t}(t, s) \leq 4(1-s):=\phi_{11}(s)
$$

and for $(t, s) \in\left[\frac{1}{3}, \frac{1}{2}\right] \times[0,1]$, it follows that

$$
c_{10}=\frac{1}{90}, \quad c_{11}=\frac{3}{4}
$$


and

$$
G_{1}(t, s) \geq c_{10} \phi_{10}(s)=\frac{1}{9} s(1-s), \quad \frac{\partial G_{1}}{\partial t}(t, s) \geq c_{11} \phi_{11}(s)=3(1-s),
$$

and for $G_{2}$, taking

$$
\phi_{20}(s)=2, \quad c_{20}=\frac{1}{20}, \quad \phi_{21}(s)=\frac{5}{2}, \quad c_{21}=\frac{1}{5},
$$

showing that

$$
G_{2}(t, s) \geq c_{20} \phi_{20}(s)=\frac{1}{10}, \quad \frac{\partial G_{2}}{\partial t}(t, s) \geq c_{21} \phi_{21}(s)=\frac{1}{2} .
$$

Condition (A3) is satisfied as the four integrals referred to are trivially positive.

For $j=0,1$, we have

$$
\begin{aligned}
& \limsup _{u_{1}^{(j)}, u_{2}^{(j)} \rightarrow 0} \max _{t \in[0,1]} \frac{\left(e^{-\left(u_{2}^{\prime}(t)+u_{1}(t)\right)^{2}}+\sqrt{\left|u_{1}^{\prime}(t)+u_{2}(t)\right|}\right.}{\max \left\{\left|u_{1}^{(j)}\right|,\left|u_{2}^{(j)}\right|\right\}}=+\infty, \\
& \liminf _{u_{1}^{(j)}, u_{2}^{(j)} \rightarrow+\infty} \min _{t \in[0,1]} \frac{\left(e^{-\left(u_{2}^{\prime}(t)+u_{1}(t)\right)^{2}}+\sqrt{\left|u_{1}^{\prime}(t)+u_{2}(t)\right|}\right)}{\max \left\{\left|u_{1}^{(j)}\right|,\left|u_{2}^{(j)}\right|\right\}}=0, \\
& \liminf _{u_{1}^{(j)}, u_{2}^{(j)} \rightarrow 0} \min _{t \in[0,1]} \frac{\left(2+\cos \left(u_{2}(t)+u_{1}(t)\right)^{2}\left(\sin \left(u_{1}^{\prime}(t) u_{2}^{\prime}(t)\right)+1\right)\right.}{\max \left\{\left|u_{1}^{(j)}\right|,\left|u_{2}^{(j)}\right|\right\}}=+\infty, \\
& \limsup _{u_{1}^{(j)}, u_{2}^{(j)} \rightarrow+\infty} \max _{t \in[0,1]} \frac{\left(2+\cos \left(u_{2}(t)+u_{1}(t)\right)^{2}\left(\sin \left(u_{1}^{\prime}(t) u_{2}^{\prime}(t)\right)+1\right)\right.}{\max \left\{\left|u_{1}^{(j)}\right|,\left|u_{2}^{(j)}\right|\right\}}=0,
\end{aligned}
$$

and, therefore, conditions (B1) hold.

So, by Theorem 3 , there is at least one positive solution $(u, v) \in\left(C^{1}[0,1] \times C^{1}[0,1]\right)$ of problem (19), which is solution of (18).

\section{Acknowledgements}

Not applicable.

Funding

This work was supported by Fundação para a Ciência e Tecnologia by the project UID/MAT/04674/2019. The corresponding author is partially supported by Fundação Calouste Gulbenkian.

Availability of data and materials

Data sharing not applicable to this article as no datasets were generated or analyzed during the current study.

Competing interests

There are no competing interests.

\section{Authors' contributions}

Both authors contributed equally to the writing of this paper. Both authors read and approved the final manuscript.

\section{Author details}

${ }^{1}$ Faculdade de Ciências e Tecnologia, Núcleo de Matemática e Aplicações (NUMAT), Universidade de Cabo Verde, Praia, Cabo Verde. ${ }^{2}$ Departamento de Matemática, Escola de Ciências e Tecnologia, Centro de Investigação em Matemática e Aplicações (CIMA), Instituto de Investigação e Formação Avançada, Universidade de Évora, Évora, Portugal.

\section{Publisher's Note}

Springer Nature remains neutral with regard to jurisdictional claims in published maps and institutional affiliations. 


\section{References}

1. Abdolmaleki, E., Najafi, H.: An efficient algorithmic method to solve Hammerstein integral equations and application to a functional differential equation. Adv. Mech. Eng. 9(6), 1-8 (2017). https://doi.org/10.1177/1687814017701704

2. Agarwal, R., O'Regan, D., Wong, P.: Constant-Sign Solutions of Systems of Integral Equations. Springer, Basel (2013)

3. Almousa, M., Ismail, A.: Numerical solution of Fredholm-Hammerstein integral equations by using optimal homotopy asymptotic method and homotopy perturbation method. In: AIP Conference Proceedings, vol. 1605, p. 90 (2014). https://doi.org/10.1063/1.4887570

4. Atkinson, K.: The Numerical Solution of Integral Equations of the Second Kind. Cambridge Monographs on Applied and Computational Mechanics. New York (1997)

5. Bakodah, H., Darwish, M.: Solving Hammerstein type integral equation by new discrete Adomian decomposition methods. Math. Probl. Eng. 2013, Article ID 760515 (2013). https://doi.org/10.1155/2013/760515

6. Barzkar, A., Assari, P., Mehrpouya, M.: Application of the CAS wavelet in solving Fredholm-Hammerstein integral equations of the second kind with error analysis. World Appl. Sci. J. 18(12), 1695-1704 (2012). https://doi.org/10.5829/idosi.wasj.2012.18.12.467

7. Bica, A., Curila, M., Curila, S.: About a numerical method of successive interpolations for functional Hammerstein integral equations. J. Comput. Appl. Math. 236, 2005-2024 (2012)

8. Cabada, A., Cid, J., Infante, G.: A positive fixed point theorem with applications to systems of Hammerstein integral equations. Bound. Value Probl. 2014, 254 (2014). https://doi.org/10.1186/s13661-014-0254-8

9. Cardinali, T., Papageorgiou, N.: Hammerstein integral inclusions in reflexive Banach spaces. Proc. Am. Math. Soc. 127(1), 95-103 (1999)

10. Cianciaruso, F., Infante, G., Pietramala, P.: Solutions of perturbed Hammerstein integral equations with applications. Nonlinear Anal., Real World Appl. 33, 317-347 (2017)

11. Coclite, M.: Positive solutions of Hammerstein integral equation with singular nonlinear term. Topol. Methods Nonlinear Anal. 15, 235-250 (2000)

12. Cui, Y., Sun, J.: On existence of positive solutions of coupled integral boundary value problems for a nonlinear singular superlinear differential system. Electron. J. Qual. Theory Differ. Equ. 41, 1 (2012)

13. Faraci, F., Moroz, V:: Solutions of Hammerstein integral equations via variational principle. J. Integral Equ. Appl. 15(4), 385-402 (2003)

14. Farengo, R., Lee, Y., Guzdar, P.: An electromagnetic integral equation: application to microtearing modes. Phys. Fluids 26, 3515 (1983). https://doi.org/10.1063/1.864112

15. Graef, J., Kong, L., Minhós, F.: Generalized Hammerstein equations and applications. Results Math. 72, 369-383 (2017)

16. Guo, D., Lakshmikantham, V.: Nonlinear Problems in Abstract Cones. Academic Press, San Diego (1988)

17. Hammerstein, A.: Nichtlineare intergralgleichungen nebst anwendungen. Acta Math. 54, 117-176 (1929)

18. Infante, G., Minhós, F:: Nontrivial solutions of systems of Hammerstein integral equations with firsrt derivative dependence. Mediterr. J. Math. 14, 242 (2017). https://doi.org/10.1007/s00009-017-1044-1

19. Kurz, L., Nowosad, P., Saltzberg, B.R.: On the solution of a quadratic integral equation arising in signal design. J. Franklin Inst. 281, 437-454 (1966)

20. Minhós, F., de Sousa, R.: On the solvability of third-order three points systems of differential equations with dependence on the first derivatives. Bull. Braz. Math. Soc. (2017). https://doi.org/10.1007/s00574-016-0025-5

21. Sauter, S., Schwab, C.: Boundary Element Methods. Springer, Berlin (2011)

22. Shahsavaran, A.: Lagrange functions method for solving nonlinear Hammerstein Fredholm-Volterra integral equations. Appl. Math. Sci. 5(49), 2443-2450 (2011)

23. Xu, J., Yang, Z:: Positive solutions for a system of nonlinear Hammerstein integral equations and applications. J. Integral Equ. Appl. 24(1), 131-147 (2012)

24. Yang, Z., Zhang, Z.: Positive solutions for a system of nonlinear singular Hammerstein integral equations via nonnegative matrices and applications. Positivity 16(4), 783-800 (2012). https://doi.org/10.1007/s11117-011-0146-4

25. Zarebnia, M., Khani, S.: Numerical solution of Hammerstein integral equations by using quasi-interpolation. Int. J. Math. Comput. Sci. 7(4), 716-719 (2013)

26. Zhang, X., Liu, L., Wu, Y.: Existence and uniqueness of iterative positive solutions for singular Hammerstein integral equations. J. Nonlinear Sci. Appl. 10, 3364-3380 (2017)

\section{Submit your manuscript to a SpringerOpen ${ }^{\circ}$ journal and benefit from:}

- Convenient online submission

- Rigorous peer review

- Open access: articles freely available online

- High visibility within the field

- Retaining the copyright to your article

Submit your next manuscript at $\gg$ springeropen.com 